msh-mss Mathématiques et sciences humaines

189 | Printemps 2010

Varia

\title{
Francis Galton ou comment photographier une moyenne
}

Francis Galton's composite portraits: the picture of a number

Simona Morini

\section{(2) OpenEdition}

1 Journals

Édition électronique

URL : http://journals.openedition.org/msh/11633

DOI : $10.4000 / \mathrm{msh} .11633$

ISSN : 1950-6821

Éditeur

Centre d'analyse et de mathématique sociales de l'EHESS

Édition imprimée

Date de publication : 5 janvier 2010

Pagination : 5-17

ISSN : 0987-6936

\section{Référence électronique}

Simona Morini, «Francis Galton ou comment photographier une moyenne », Mathématiques et sciences humaines [En ligne], 189 | Printemps 2010, mis en ligne le 22 juin 2010, consulté le 23 juillet 2020. URL : http://journals.openedition.org/msh/11633 ; DOI : https://doi.org/10.4000/msh.11633

(ㄷ École des hautes études en sciences sociales 


\title{
FRANCIS GALTON OU COMMENT PHOTOGRAPHIER UNE MOYENNE
}

\author{
Simona MORINI ${ }^{1}$
}

RÉSUMÉ - À travers la réalisation d' « images génériques» ou «photos composées 》(des images obtenues en superposant plusieurs photos du même individu, ou bien d'individus appartenant à un même "genre ») Francis Galton essayait de saisir l'image de quelque chose d'abstrait : le genre, la moyenne statistique, l'«essence» d'un individu ou le caractère typique d'un groupe. Il espérait éliminer la subjectivité de ce qui est le plus typiquement subjectif : la formation des images mentales, des concepts abstraits. Après quelques mois, il abandonna l'idée que certains traits du visage puissent être mis en relation avec un " type " (le composé d'un "criminel moyen "était identique au composé d'un "pauvre moyen ») et il se rendit compte que la loi des erreurs, fondée sur la moyenne arithmétique, tout en étant très utile aux statisticiens pour la description des phénomènes de la vie et de la société, peut dans beaucoup de cas "conduire à des absurdités quand les phénomènes présentent de fortes déviations par rapport à la norme ». Galton n'a pas développé cette intuition, mais comme cela lui arrivait assez. souvent, il a su montrer la voie pour dépasser ses propres théories.

MOTS-CLÉS - Galton, Homme moyen, Images mentales, Mesure, Photographie, Statistique, Visualisation

SUMMARY - Francis Galton's composite portraits: the picture of a number

By realising "generic images" or “composite portraits" (images obtained by superimposing several pictures of the same individual or of different individuals belonging to the same family or group) Francis Galton was trying to capture the image of an abstract entity: a type, a statistical average, the "essence" of an individual or the "typical" character of a group. He was trying to eliminate subjectivity from what is usually considered characteristically subjective: the formation of mental images, of abstract concepts. After a few months he had to admit that the idea that certain physical traits could be related to a "type" was wrong (the composite of an "average criminal" is very much the same as the composite of an "average poor person") and he realized that the law of errors based on an arithmetic average, though useful for statisticians trying to describe the phenomena of life and society, could lead in many cases to "absurdities when the phenomena show significant deviations from normality". Galton didn't develop this intuition further but, as was often the case for him, he paved the way for going beyond his own theories. Visualisation

KEYWORDS - Average man, Galton, Measure, Mental images, Photography, Statistics,

Le 25 avril 1879, au cours des rencontres du vendredi soir de la Royal Institution, dans la même salle où en 1839 avait été annoncée l'invention de la photographie, Sir Francis Galton, neveu d'Erasmus Darwin et cousin de Charles Darwin, explorateur de la Namibie et président de la prestigieuse Royal Geographical Society - un prototype du savant victorien à succès - présentait au public les premiers exemplaires de ses «!portraits génériques!» ou «!photos composées!» : des images obtenues en superposant plusieurs

1 Università IUAV di Venezia, Convento delle Terese, Dorsoduro 2206, 30123 Venezia, simona.morini@gmail.com 
photos du même individu, ou bien d'individus appartenant au même «!genre!», c'est-à-dire «! une collection d'individus qui ont beaucoup en commun et chez lesquels les caractéristiques moyennes sont beaucoup plus fréquentes que les extrêmes! $»^{2}$.

Devant les yeux d'un public intrigué, trois lanternes magiques projetaient une image ambiguë, ni homme ni femme, obtenue en superposant les photos de trois frères et sœur -!deux garçons et une femme. Bien que le choix des sujets (hétérogènes, quant au sexe, et photographiés avec des expressions légèrement différentes) "mettait à l'épreuve les possibilités du procédé $»^{3}$ le plus adapté à associer des «types du même genre », l'image produite était celle d'un visage plus régulier et plus agréable de chacun des visages qui la composaient et montrait de façon très claire les traits de famille communs à la fratrie. Le conférencier soulignait aussi qu'autour du visage composé des «!fantômes de traits masculins et féminins des originaux $\gg^{4}$ restaient des traces de leur diversité, qui cependant étaient tellement faibles qu'elles n'arrivaient pas à influencer la perception de la figure moyenne.

Quelques années après, présentant la photo composée de cinq garçons juifs, il fit remarquer la persistance de la boucle de cheveux « brune et nettement définie!» de l'un d'entre eux, bien que l'intensité de l'original eût été réduite par cinq au cours du processus. En superposant ce composé à celui d'un autre constitué de cinq jeunes juifs et de trois autres portraits, il restait dans l'image obtenue une faible trace de la boucle, bien que l'intensité avait été réduite treize fois, mais Galton observa que si la différence avait été moins marquée, on ne l'aurait pas notée ou elle aurait été prise pour une ombre ou une tache fortuite!: dans le composé, les différences ont tendance à disparaître et seuls les traits communs ressortent.

Après la projection au moyen des lanternes magiques, Galton invitait le public à confronter l'image qu'il venait de présenter à celle composée de trois frères et soeur, obtenue en utilisant une technique différente qui opère " comme les souvenirs qui se superposent dans l'esprit $»^{5}$ c'est à dire en superposant trois images transparentes devant l'objectif de la caméra et en les enlevant l'une après l'autre, de façon à imprimer sur la même plaque une succession de portraits différents dont le résultat final était le composé.

En expérimentateur méticuleux qu'il était, Galton avait ainsi montré que deux techniques différentes produisaient le même résultat ${ }^{6}$ et que l'utilisation de la photographie était donc fiable. Il montra alors une autre série de photos composées, obtenues en superposant les photos d'anciennes monnaies, gardées au British Museum, qui représentaient Alexandre (6 composants), Antioche, roi de Syrie (6), Démétrios (6), Cléopâtre (5), Néron (11), une combinaison de cinq différents visages de femmes grecques et une «!combinaison particulièrement belle de six différentes femmes romaines, qui produit un charmant profil idéal!» 7 .

La série suivante était moins «!classique!» et montrait les portraits composés de délinquants ordinaires : assassins, criminels, voleurs, auteurs de massacres et de toutes

\footnotetext{
${ }^{2}$ F. Galton [1879(a)], p. 162. La définition de genre et de «!portrait générique!» reprend explicitement «!!'heureuse expression du Professeur Huxley» ("the happy phrase of Professor Huxley"). Thomas Henry Huxley, président de la Royal Society et mieux connu comme le «!bulldog de Darwin!», avait écrit un traité sur David Hume, dans lequel il distingue entre les impressions génériques et celles qui sont spécifiques, en les comparant explicitement aux photos composées. T.H Huxley [1878], Ch. IV, §111.

${ }^{3}$ Ibid., p. 163.

${ }^{4}$ Ibid.

5 Ibid.

${ }^{6}$ Il illustra, dans son exposé, d'autres méthodes qu'il avait utilisées pour produire des photos composées, afin de s'assurer que le moyen par lequel elles avaient été obtenues n'avait pas d'influence sur le résultat.

${ }^{7}$ F. Galton [1879(a)], p. 165.
} 
sortes d'actes violents. À propos de ces derniers, Galton faisait remarquer au public l'existence de deux types différents!: certains du type Henry VIII, «!gros et massifs!», d'autres plus fins et minces, avec un visage différent de celui de l'Anglais quelconque. Parmi ces deux ensembles très différents d'individus, trois portraits composés donnaient l'impression d'être des portraits de frères, tant ils étaient semblables, et une fois combinés et projetés par les lanternes magiques ils produisaient un certain «!genre!» de criminel!: «!le criminel moyen!».

Pour le public savant de la Royal Institution, l'«!homme moyen!», qui avait été le protagoniste de la «!physique sociale!» d'Adolphe Quételet, était une vieille connaissance. Dans un de ses premiers écrits, ${ }^{8}$ où il analysait les caractéristiques physiques (surtout la taille et le poids) de certains groupes de personnes (soldats ou citoyens ordinaires), Quételet comparait l'homme moyen au centre de gravité des corps: il s'agissait d'un «!être fictif!» dont les caractéristiques se conforment à la moyenne des résultats obtenus pour la société toute entière, c'est-à-dire au groupe auquel il appartient. Dans un mémoire ultérieur ${ }^{9}$, où il utilise pour la première fois le terme «!homme moyen!» on lit que «!si l'homme moyen était déterminé pour une nation, il représenterait cette nation ; s'il était déterminé pour l'ensemble de l'humanité, il représenterait le type de l'espèce humaine dans son ensemble!». À partir de ses mesures des thorax des soldats écossais, il arriva à la conclusion qu'il existe un type d'homme dont les individus singuliers constituent des variations dont l'entité peut être calculée a priori et représentée par la courbe normale, gaussienne, ou « en cloche ».

Quételet interprétait les mesures concentrées autour de la partie centrale de la courbe de Gauss comme une zone de normalité, une sorte de «!centre de gravité!» social, une norme naturelle de ce qui est bien ou beau, et il comparait les différences individuelles à l'«!erreur mathématique!», à des «!perturbations momentanées!» qui obéissaient à ce qu'il appelait la «!loi des causes accidentelles!». Le type est donc déterminé par l'action de causes constantes, la variation par les causes accidentelles ou «!perturbations!». Nous savons que Quételet appliqua ensuite ses idées sur les caractéristiques physiques aux qualités morales et intellectuelles des hommes!: de la tendance au suicide à la criminalité, en faisant de sa courbe une «!loi générale qui domine l'univers!». C'était le cœur de sa «!physique sociale!».

L'homme moyen intéressait Quételet parce qu'il était plus facile à analyser que des individus réels. Il observait que tandis qu'il est très difficile d'arriver à comprendre les raisons personnelles, morales ou sociales pour lesquelles un individu suit un certain type de comportement anormal (il se tue ou tue quelqu'un d'autre, ou se comporte de façon antisociale), l'homme moyen, dont les caractéristiques sont définies par les données statistiques, est parfaitement compréhensible. Dans la moyenne, les traits individuels et exceptionnels s'effacent (exactement comme la boucle indisciplinée du garçon juif) et ce qui ressort finalement ce sont les caractéristiques d'un «!groupe!» (la «!tendance!»au suicide, à la criminalité, à l'éversion, etc. d'une communauté) qui dépendent de circonstances (les causes constantes) qui, une fois individualisées, mesurées et analysées scientifiquement, peuvent être éventuellement corrigées.

Nous avons dit que Quételet voyait dans l'homme moyen le «!type!» d'une nation, mais qu'il ne l'interprétait pas, philosophiquement, de façon, pour ainsi dire, «!essentialiste!». Comme Galton, il remarquait que, dans la moyenne, les différences s'effacent. Mais ce qu'il voyait, une fois éliminées les différences, ce n'étaient pas des essences ou des types abstraits, mais des tendances sociales réglées par des lois.

\footnotetext{
${ }^{8}$ A. Quételet [1831].

${ }^{9}$ A. Quételet [1832].
} 
Plus grand est le nombre des individus observés, plus les caractéristiques individuelles, physiques ou morales, ont tendance à disparaître et laissent voir les faits généraux en vertu desquels la société existe et se maintient ${ }^{10}$.

À partir des mesures et des données statistiques, il pouvait obtenir, par exemple, les caractéristiques d'un Belge typique (duquel les autres Belges pouvaient différer seulement de façon marginale) ; mais le même Belge rentrait aussi dans d'autres moyennes, associées à des catégories plus ou moins grandes : bruxellois, caucasien, père, ouvrier, mâle, être humain, etc.

Ce qui était intéressant pour Quételet - et pour les statisticiens de la première moitié du $\mathrm{XIX}^{\mathrm{e}}$ siècle - ce n'était pas de classer les personnes, en les divisant en catégories, mais plutôt le fait que des actions individuelles totalement chaotiques et inexplicables pouvaient donner lieu, sur une grande échelle, à des phénomènes absolument réguliers, à des véritables lois. Le fait surprenant était que dans telle ville, région ou État, le nombre des lettres non remises, de suicides, de crimes, de mariages et d'autre actions apparemment «!libres!» et imprévisibles était presque constant. L'homme moyen, donc, tout en étant totalement abstrait, créé par des données et des opérations numériques, avait l'air d'être plus «!réel!» que l'ensemble chaotique et incompréhensible des hommes réels qu'ils considéraient comme des victimes passives, plus que des protagonistes de l'action sociale. Les implications politiques sont assez claires et ont été développées de façon plus sophistiquée par Karl Marx!: pour changer le monde on ne doit pas intervenir sur les individus, mais sur la société qui en détermine les comportements.

Galton n'était pas un enthousiaste de la «!physique sociale!» de Quételet et de ses lois. «!Certains démocrates résolus regardent peut-être avec intérêt une foule d'hommes médiocres, mais, pour d'autres personnes, ils ne sont pas intéressants du tout!» ${ }^{11}$. Il manifestait un mépris aristocratique pour tout ce qui entrait dans la moyenne et il était même ironique à cet égard :

C'est difficile de comprendre pourquoi les statisticiens limitent leurs enquêtes aux moyennes et ne s'abandonnent pas à des visions plus larges. Leur âme semble être aussi sourde à l'appel de la variété que celle d'un de nos concitoyens, né dans une des régions les plus plates de l'Angleterre, dont le souvenir de la Suisse était que, si on avait pu jeter les montagnes dans les lacs, l'on aurait pu se libérer de deux ennuis d'un seul coup ${ }^{12}$.

D'après la théorie de Darwin, ce qui paraissait intéressant, aux yeux des savants, c'était surtout la variation et Galton, qui était conscient d'appartenir à une famille de génies, concentra son attention sur la transmission et l'héritabilité des traits exceptionnels ${ }^{13}$. Il ne considérait pas les extrémités de la courbe normale ou gaussienne, comme des erreurs ou des imperfections, mais comme les seuls cas intéressants à étudier.

Galton n'arriva jamais à expliquer l'héritabilité du talent ou de l'intelligence (pas dans la direction qu'il espérait, tout au moins). Ses recherches (sur les graines de haricots) montraient que la distribution d'un caractère donné dans une population ne variait pas d'une génération à l'autre et que même en croisant seulement les éléments exceptionnels de la population (les graines les plus lourdes, par exemple), les descendants avaient tendance, sur la longue durée, à régresser vers la moyenne de la population initiale. Mais il arriva quand même à démontrer que le coefficient de réversion valait comme relation entre deux variables convenablement choisies et était donc indépendant des lois de l'hérédité!: une simple propriété des opérations statistiques, en général. Pour le distinguer de la

\footnotetext{
10 A. Quételet [1842], p. 6.

${ }^{11}$ F. Galton [1889(a)], p. 407.

${ }^{12}$ F. Galton [1889], p. 62.

13 F. Galton [1869].
} 
réversion, il l'appela «!coefficient de régression!», un cas particulier de la corrélation statistique qui mesurait le degré de dépendance des variables l'une par rapport à l'autre.

Les historiens ont justement souligné l'importance que Galton attribuait à la diversité et ont observé que l'élément plus révolutionnaire de son oeuvre fut d'ouvrir la voie à la notion de loi statistique, tout en se libérant du déterminisme implicite dans la conception des lois qui était celle de Quételet et des statisticiens de la première moitié du $\mathrm{XIX}^{\mathrm{e}}$ siècle. Mais je ne m'intéresse pas ici à cette différence fondamentale qui a déjà été très bien soulignée par les historiens.

Le cas des photos composées de Galton n'est pas seulement intéressant pour l'essentialisme issu de la psychologie qui l'anime, mais surtout parce qu'il montre très clairement les mécanismes de «!création de réalité!» liés à un certain usage des images et de la photographie et dominés par une conception de l'objectivité et par les valeurs de la mesure et de la précision que Galton poursuivait de façon obsessionnelle et maniaque. L'élégant sir Francis, pendant ses voyages, mesurait avec un sextant - pour des raisons statistiques, bien sûr! - les formes des femmes africaines. Aussi, en essayant de dessiner une «!carte de la beauté!» des îles britanniques, il classait les filles qu'il rencontrait dans la rue comme «!séduisantes, indifférentes ou repoussantes!» en utilisant «! une aiguille montée comme un poinçon pour faire des trous dans un morceau de papier en forme de croix!» qu'il tenait dans sa poche. Dans la partie supérieure de la croix, il «!marquait!» les belles qu'il croisait sur sa route, sur la cote horizontale les «!moyennes!» et dans la partie inférieure les «!repoussantes!» 14 .

Il mesura et quantifia n'importe quoi!: de l'ennui d'une conférence, mesuré en observant les mouvements de certaines parties du corps dans le public, à la dignité des peuples ou l'excitation des personnes pendant les courses en observant la rougeur de leurs visages. Et aussi, évidemment, têtes, crânes et toute partie possible du corps. Dans l'enceinte de 1'Exposition Internationale de 1884, il installa un laboratoire où contre 3 pennies on était mesuré de la tête aux pieds -!corps et âme!- et où l'on obtenait aussi une évaluation finale. Plus tard, il transplanta cette activité, qui dura plusieurs années, dans un Laboratoire d'Anthropométrie, au musée de South Kensington qui devint le coeur de ses programmes d'eugénique. La vision scientifique de l'époque «!accordait du mérite à la fiabilité méticuleuse du bourgeois plus qu'à la vivacité du génie!» et s'accompagnait d'une vision moralisatrice, d'une éthique du travail précis et sans relâche, à l'intérieur de laquelle le contrôle de soi dominait les «!tentations de la chair et de l'esprit », c'est-à-dire la subjectivité15. Si en effet la deuxième moitié du XIX ${ }^{\mathrm{e}}$ siècle a été caractérisée par la séduction des nombres, Sir Francis était l'un des plus typiques et des plus influents représentants de cette séduction ou manie. Comme l'écrivait Stephen Jay Gould «!la quantification était le dieu de Galton et, à sa droite, on pouvait trouver la solide croyance en l'héritabilité de tout ce que le savant pouvait mesurer!»16.

Mais revenons à la salle de la Royal Institution. Le choix des sujets est en soi significatif de la prédilection de Galton pour les cas exceptionnels. D'un côté, des exemples de génialité et de perfection, inspirés surtout d'un idéal typique de son époque!: le classicisme. Il suffit d'avoir visité la maison de Sir John Soane à Londres pour comprendre le charme qu'exerçaient encore les monde grec et romain sur les hommes du $\mathrm{XIX}^{\mathrm{e}}$ siècle. Dans les traités de physionomie et de craniométrie de l'époque l'on voit très bien que les traits du visage et des corps grecs représentaient la perfection, le plus haut niveau de développement de l'esprit humain!: «!le nez grec, avec son pont élevé, coïncide

\footnotetext{
14 F. Galton [1909], p. 315-316.

15 Pour une reconstruction de cette vision de la science, cf. L. Daston et P. Galison [1992], p. 83.

16 S.J. Gould [1981], trad.it, p. 89.
} 
non seulement avec la beauté esthétique, mais aussi avec la perfection évolutive!» ${ }^{17}$. Par contre, au niveau plus bas de l'échelle de l'humanité - de l'autre côté de la gaussienne l'on pouvait trouver, pas trop loin des animaux et des singes, avec lesquels les craniométriques de l'époque soulignaient sans cesse les similarités, les criminels et les déviants en général. L'intérêt morbide de l'époque victorienne pour la criminalité était étroitement lié à une sorte de peur d'une décadence de la société européenne qui détermina ensuite le succès de l'eugénique!; Galton avait reçu des centaines de photos de criminels du directeur d'un pénitencier qui souhaitait mettre au point un plan scientifique contre le crime, à partir desquelles il avait obtenu ses composés.

À l'époque où Galton faisait sa conférence, il était un disciple assez convaincu -!comme la plupart de ses contemporains!- sur la physiognomie et pensait que l'analyse des visages -!et des composés en particulier!- pouvait révéler une forte corrélation entre les traits du visage et les caractéristiques mentales et psychologiques -!parmi lesquelles beaucoup étaient considérées comme héréditaires!- de certains types d'individus. Plus tard, il se rendit compte -!justement à travers l'analyse des composés des criminels!- que la criminalité était une mentalité plus qu'une tare héréditaire, et que le composé d'un criminel moyen était identique au composé d'un pauvre moyen ou d'un individu «!typique appartenant aux classes plus basses!» ${ }^{18}$. Il abandonna alors l'idée que certains traits du visage puissent être mis en relation avec un «!type!» de criminel et se réfuta explicitement lui-même - lainsi que les lombrosiens.

Mais à cette période-là, ce n'était pas exactement ce qui le passionnait vraiment. C'est son élève et biographe Karl Pearson, le célèbre «!fondateur!» de la statistique, qui nous dit qu' à partir de la moitié des années soixante-dix de son siècle, les intérêts scientifiques de Galton s'orientèrent surtout vers le côté psychométrique de l'anthropologie. Et bien qu'il n'ait jamais complètement abandonné la physionomie et la croyance selon laquelle la dimension du crâne était en quelque manière corrélée avec l'intelligence, il modifia et abandonna avec le temps l'idée que les caractères anthropométriques puissent révéler quelque chose sur le fonctionnement mental ${ }^{19}$. Il se concentra alors sur l'étude des fonctions mentales et sur leur mesure directe, au point que Pearson et beaucoup de ses contemporains le considéraient comme un pionnier de la psychologie expérimentale et estimaient que ses techniques de recherche étaient très avancées par rapport à celles de l'école allemande qui à cette époque était très «!à la mode!».

Ce qui pour Galton était absolument extraordinaire et presque «!magique!» dans les composés était qu'ils étaient des «!photos!» de quelque chose d'abstrait!: le genre, la moyenne statistique, l'«!essence!» d'un individu ou le caractère typique d'un groupe. Tous ces termes ont un air de famille (il s'agit de différents aspects d'un processus d'abstraction et de standardisation des phénomènes et des personnes) mais ils ne sont pas exactement synonymes et sont ontologiquement nuancés. De même, il est important distinguer le typique de l'idéal (qui exprime la perfection d'un type) et de la caractéristique (qui incarne le type d'un individu particulier). Tous ces concepts font partie du problème de saisir dans une image (que ce soit un tableau, un dessin, une photo), dans un concept ou un nombre, une classe entière de phénomènes ou de caractéristiques.

Chacun d'eux implique des ontologies (et des esthétiques) qui peuvent être très différentes, comme le montre la comparaison entre les oeuvres graphiques des anatomistes du XVIII ${ }^{\mathrm{e}}$ siècle étudiées par Lorraine Daston et Peter Galison!:

\footnotetext{
${ }^{17}$ Cope [1887] ; cité dans Gould [1981], trad.it., p. 121.

18 F. Galton [1883], p. 15.

${ }^{19}$ K. Pearson [1924], p. 211.
} 
For Goethe, the depiction of the Typus did represent something in nature (though not apparent from this or that individual). For Albinus [professeur d'anatomie à Leyden, moitié du XVIII ${ }^{\mathrm{e}}$ siècle], the " true» representation of a subject referred to nature not only because it borrowed from several individuals but because it improved [«ideally»] above any single one of them. For Hunter [anatomiste britannique, deuxième moitié du XVIII ${ }^{\mathrm{e}}$ siècle], the link to the general occurred through a particular individual, chosen precisely that it might represent (in both senses) a whole class: different as they were, all three views took it for granted that a single representation could stand in for a myriad of variations found in nature ${ }^{20}$.

Par rapport à ces premières versions de l'exigence d'être «!fidèles à la nature!», la photographie peut être considérée comme la réalisation d'un idéal épistémologique. Plus tard on se rendit compte que la photographie était aussi «!subjective!», même si c'était d'une façon différente de la peinture, et donc qu'elle avait seulement fait reculer et modifié - et non pas résolu - le problème de l'objectivité. Aux yeux de Galton tout de même, l'usage de la photographie rendait possible un vrai miracle!: éliminer la subjectivité!; non seulement de la représentation du réel, mais aussi de ce qui est le plus typiquement subjectif!: la formation des images mentales, des concepts abstraits. Ce qui apparait de façon floue et indistincte aux yeux de l'esprit (par exemple quand on imagine de façon générique un animal, une figure géométrique ou un type d'objet) devient clairement défini et visible dans le portrait générique (ou composé). Il représente ainsi

\begin{abstract}
a perfect example... of what the metaphysicians mean by generalisations, when the objects generalized are objects of vision, and when they belong to the same typical group, one important characteristic of which is that medium characteristics should be far more frequent than divergent ones ${ }^{21}$.
\end{abstract}

Il s'étonnait aussi - lavec une ingénuité toute positiviste!- de la certitude avec laquelle les métaphysiciens affirmaient que les généralisations étaient impossibles et du fait qu'ils considéraient l'idée même comme absurde. La photographie, en effet, arrivait selon lui à montrer ce que les mots n'arrivaient pas à décrire.

Il existe donc un lien étroit entre ces photos et les études de psychologie que Galton faisait à cette époque sur les «!images génériques!». Galton voyait dans le processus de formation d'une photo composée l'équivalent «!objectif!»-!et donc «!parfait!»!- de l'opération mentale subjective -let donc imparfaite!- par laquelle nous nous formons des impressions complexes et nous imaginons des concepts abstraits. L'idée qu'il avait dans la tête était tout à fait semblable à celle décrite par Kant dans un long passage de la Critique du jugement!:

Il faut noter - écrit Kant - que non seulement, d'une façon que nous
n'arrivons pas à concevoir, l'imagination peut rappeler à l'occasion les
signes des concepts même après une longue période de temps, mais qu'elle
peut aussi reproduire l'image et la figure d'un objet parmi un nombre infini
d'objets d'espèce différente, ou aussi de la même espèce. De plus, quand
notre esprit fait des comparaisons, selon toute vraisemblance et bien que la
conscience n'en soit pas suffisamment avertie, il peut presque superposer
une image sur l'autre et tirer de la congruence de celles de même espèce une
moyenne qui sert de mesure commune à toutes. Quelqu'un a vu mille
personnes adultes. S'il veut procéder par comparaison [à l'estimation] de la
grandeur moyenne de l'homme, son imagination (comme je le crois) va faire
coüncider un grand nombre de figures (peut-être les mille). Et, s'il m'est
permis d'utiliser ici l'analogie avec la représentation optique, c'est dans
l'espace où le plus grand nombre de choses se réunissent, et à l'intérieur du
contour où l'espace est éclairé par les couleurs les plus vives, qu'il va

20 L. Daston, P.Galison [1992], p. 107.

21 F. Galton [1879(c)], p. 163. 
reconnaître la taille moyenne, qui est également éloigné - tant en hauteur qu'en largeur - des limites extrêmes des tailles plus grandes et plus petites. Et c'est bien là, la taille d'un homme beau 22 .

Je n'ai pas de preuve que Galton connaissait ce passage, ni Kant d'une façon générale, mais il avait écrit un essai sur les images génériques qui faisait référence à une psychologie de d'inspiration huméenne, telle que celle développée par Thomas Huxley dans son livre sur Hume, que Galton citait explicitement dans son essai. Les idées simples étaient considérées comme des copies des impressions simples, tandis que les idées ou impressions complexes se produisaient en superposant dans la mémoire les copies des idées simples. La conclusion de Huxley, évidemment, était fortement anti-nominaliste!: le caractère inachevé des impressions spécifiques est d'un autre type que celui des impressions génériques (les impressions complexes formées en superposant plusieurs impressions, plus ou moins semblables), et donc celles-ci «!ne peuvent pas être considérées comme des copies, dans le sens strict du terme, de ces impressions, et en même temps peuvent exister dans l'esprit indépendamment du langage!» ${ }^{23}$. Le processus mental envisagé par Galton était exactement le même que celui décrit par Kant, mais Galton en soulignait l'imperfection. La mémoire reproduit des copies imprécises par rapport à l'original et chaque nouvelle impression montre quelque chose que nous avions oublié. Les souvenirs sont donc des «!esquisses, plus que des portraits!» ${ }^{24}$, de l'original. Quand les impressions se superposent dans l'esprit, la mémoire «!fixe!» les caractéristiques saillantes d'un objet tandis que les caractéristiques secondaires sont négligées et mises de côté.

C'est exactement ce qui se passe quand on superpose les photographies!: les points où les visages présentent des éléments communs sont renforcés et évidents dans l'image composée («!à l'intérieur du contour où l'espace est éclairé par les couleurs plus vives!» comme dit Kant), tandis que les points où ils diffèrent restent vagues et «!estompés!». «!Une image mentale générique n'est qu'un portrait générique imprimé dans le cerveau par les impressions successives de ses images composantes!» ${ }^{25}$. C'est «!laisser tomber une image sur l'autre!», mais -!ajoutait Galton!- la photographie, à la différence de l'homme, a une mémoire parfaite!: elle ne fait pas de fautes.

Dans un essai précédent Galton avait écrit que

A composite portrait represents the picture that would rise before the mind's eye of an individual who had the gift of pictorial imagination in an exalted degree ${ }^{26}$.

Au cours de ses recherches en psychologie, Galton avait plusieurs fois observé que la capacité de visualisation (ou imagination picturale) varie d'une personne à l'autre. Dans une de ses plus célèbres expériences de psychologie, il demanda à des centaines de personnes de décrire leur déjeuner du matin, en spécifiant les objets, la nourriture, les couleurs, la disposition et d'autres détails très précis. Tandis que certaines personnes avaient des souvenirs très imprécis, partiels et confus, d'autres se rappelaient les moindres détails. Galton observait à ce propos que plus cette capacité est développée, plus il est facile pour le cerveau de produire des «!blended memories!», c'est-à-dire de composer, superposer les souvenirs jusqu'à former une image unique qui en est une version affaiblie et souvent défectueuse : l'impression générale, justement. Il observa aussi que la capacité de visualisation est présente au degré maximum chez les génies mathématiques, les poètes

22 I. Kant, Critique du Jugement, section I, Livre I, $\$ 17$.

23 T. Huxley [1878], ch.IV, p. 113.

24 Ibid., p. 112.

${ }^{25}$ F. Galton [1879(a)], p. 166.

26 F. Galton [1878], p. 97. 
et chez les artistes en général et il arriva même à définir la spécificité de l'art comme «!la capacité de produire des images qui ne sont copies d'aucun individu, mais qui représentent les caractéristiques d'une classe entière» ${ }^{27}$. Il pose alors la question!:

If a person gifted with the visualising power in perfection should pose his eye in the place of the object glass of the camera, would the generic image in his brain be identical with the photographic composite? ${ }^{28}$.

La réponse est «non » :

the imaginative power even of the highest artists is far from precise, and is so apt to be biased by special cases that may have struck their fancies, that no two artists agree in any of their typical forms. The merit of the photographic composite is its mechanical precision, being subject to no errors beyond those incidental to all photographic reproductions ${ }^{29}$.

Il faut aussi remarquer qu'un des éléments essentiels pour la réussite d'une photo composée est le temps d'exposition. Les temps d'exposition très longs créent une image plus nette -!et donc prévalente dans le composé!- par rapport à d'autres ayant des temps d'exposition plus courts. Dans le cas de l'esprit, au contraire, les cas exceptionnels laissent sur le cerveau une impression plus forte -let les cas habituels une impression plus faible!- de ce que les nombres enregistrent. L'intensité de l'effet «!subjectif!» n'est jamais proportionnelle à l'intensité de la cause «!objective!», comme le montre la loi de WeberFechner, selon laquelle plus forte est la stimulation des sens, plus faible leur pouvoir de discernement. Si une salle est éclairée par une seule bougie et qu'on en allume une deuxième, l'oeil aperçoit immédiatement la différence. Mais si les bougies sont 1000, pour noter une différence, il faudra en allumer encore 1000 et non pas une seule.

Quelque mois après, le 20 novembre 1879, dans une mémoire à la Royal Society, Galton affirma explicitement que la loi des erreurs fondée sur la moyenne arithmétique, tout en étant très utile aux statisticiens pour la description des phénomènes de la vie et de la société peut dans beaucoup de cas «!conduire à des absurdités quand les phénomènes présentent des fortes déviation par rapport à la norme!» ${ }^{30}$. À propos de la taille, par exemple, il observe que «!la loi est correcte quand les mesures sont normales, mais elle implique que l'on supprime l'existence de géants, dont la taille est plus du double de celle des hommes ordinaires ainsi que celle des nains, dont la taille est moins que rien!». ${ }^{31}$ Cette intuition de Galton est à la base de ce que Marc Barbut a appelé «!l'homme extrême!» de Pareto $^{32}$ et est aujourd'hui au centre d'une interprétation de la statistique où l'importance des moyennes dans la description des phénomènes est restreinte à un nombre assez petit de cas, les cas où justement les variations de la moyenne sont minimes (comme dans le cas des thorax de Quételet) ${ }^{33}$. Galton n'a pas beaucoup développé cette intuition mais, comme cela se passait assez souvent chez lui, il a su indiquer la voie pour dépasser ses propres théories !

\footnotetext{
${ }^{27}$ F. Galton [1879(c)], p. 160.

28 Ibid., p. 166.

${ }^{29}$ F. Galton [1879], p. 134.

30 F. Galton [1879(b)], p. 367.

31 Ibid.

32 M. Barbut [2007].

33 D. Zajdenweber [2000], B. Mandelbrot [1997].
} 


\section{BIBLIOGRAPHIE}

BARBUT M., « Homme moyen ou homme extrême ?!: de Vilfredo Pareto (1896) à Paul Levy (1936) en passant par Maurice Fréchet et quelques autres!», La mesure des inégalités, Genève, Droz, 2007, p. 147168.

COPE E.D., The Origin of the Fittest, New York, Macmillan, 1887.

DASTON L., GALISON P., “The image of objectivity”, Representations, ${ }^{\circ}$ 40, Special Issue: Seeing Science, 1992, p. 81-128.

GALTON F., Hereditary Genius: An Inquiry into Its Laws and Consequences, London, 1869.

GALTON F., "Composite portraits made by combining those of different persons into a single figure", Nature 18, May 23, 1878.

GALTON F., "Composite Portraits”, Journal of the Anthropological Institute, vol.VIII, 1879.

GALTON F., "Generic Images", Royal Institution, Weekly evening meeting, Friday, April 25, 1879(a).

GALTON F., "The geometric mean in vital and social statistics", Proceedings of the Royal Society, November 20, 1879(b).

GALTON F., "Generic images", Nineteenth century, vol 6, July, 1979(c).

GALTON F., (1883), Inquiries into Human Faculty and its Development, London, Macmillan, 1883.

GALTON F., Natural Inheritance, London, Macmillan, 1889.

GALTON F., (1889a), "President's Address", Journal of the Anthropological Institute of Great Britain and Ireland 18, 1889(a).

GALTON F., Memories of my Life, London, Methuen, 1909.

GOULD S.J., The Mismeasure of Man, [trad it.] Intelligenza e pregiudizio, Il Saggiatore, Milano, 1981.

HUXLEY T., Hume, London, 1878.

MANDELBROT B., Fractales, hasard et finance, Paris, Flammarion, 1997.

PEARSON K., The Life, Letters and Labours of Francis Galton, Cambridge,Cambridge University Press, 1924.

QUETELET A., «!Recherches sur la loi de croissance de l'homme!», Annales d'Hygiène Publique et de Mèdicine Legale 6, 1831, p. 89-113.

QUETELET A., «!Recherches sur le penchant au crime aux différents ages!», Nouveaux mémoires de l'académie royale des sciences et belles-lettres de Bruxelles, 7, 1932.

QUETELET A., A treatise of Man and the development of his faculties, trans. R. Knox, Chambers, Edinburgh, 1842.

ZAJDENWEBER D., Economie des extrêmes, Paris, Flammarion, 2000. 